\title{
A streamlined method for systematic, high resolution in situ analysis of mRNA distribution in plants Sinéad Drea ${ }^{1,2}$, Julia Corsar ${ }^{1}$, Brian Crawford ${ }^{1}$, Peter Shaw ${ }^{1}$, Liam Dolan ${ }^{1}$ and John H Doonan*1
}

\author{
Address: ${ }^{1}$ John Innes Centre, Norwich NR4 7UH, UK and 2Department of Molecular, Cellular and Developmental Biology, P.O. Box 208104, Yale \\ University, 266 Whitney Ave., New Haven, CT 06520-8104, USA \\ Email: Sinéad Drea - sinead.drea@yale.edu; Julia Corsar - jacorsar@btinternet.com; Brian Crawford - bccrawfo@biomail.ucsd.edu; \\ Peter Shaw - peter.shaw@bbsrc.ac.uk; Liam Dolan - liam.dolan@bbsrc.ac.uk; John H Doonan* - John.doonan@bbsrc.ac.uk \\ * Corresponding author
}

Published: 06 October 2005

Plant Methods 2005, I:8 doi:10.1 186/1746-48II-I-8

This article is available from: http://www.plantmethods.com/content/I/I/8

(C) 2005 Drea et al; licensee BioMed Central Ltd.

This is an Open Access article distributed under the terms of the Creative Commons Attribution License (http://creativecommons.org/licenses/by/2.0), which permits unrestricted use, distribution, and reproduction in any medium, provided the original work is properly cited.
Received: 31 August 2005

Accepted: 06 October 2005

\begin{abstract}
Background: In situ hybridisation can provide cellular, and in some cases sub-cellular, resolution of mRNA levels within multicellular organisms and is widely used to provide spatial and temporal information on gene expression. However, standard protocols are complex and laborious to implement, restricting analysis to one or a few genes at any one time. Whole-mount and reverse transcriptase-PCR (RT-PCR) based protocols increase throughput, but can compromise both specificity and resolution. With the advent of genome-wide analysis of gene expression, there is an urgent need to develop high-throughput in situ methods that also provide high resolution.
\end{abstract}

Results: Here we describe the development of a method for performing high-throughput in situ hybridisations that retains both the high resolution and the specificity of the best manual versions. This refined semi-automated protocol has the potential for determining the spatial and temporal expression patterns of hundreds of genes in parallel on a variety of tissues. We show how tissue sections can be organized on microscope slides in a manner that allows the screening of multiple probes on each slide. Slide handling, hybridisation and processing steps have been streamlined providing a capacity of at least 200 probes per week (depending on the tissue type). The technique can be applied easily to different species and tissue types, and we illustrate this with wheat seed and Arabidopsis floral meristems, siliques and seedlings.

Conclusion: The approach has the high specificity and high resolution of previous in situ methods while allowing for the analysis of several genes expression patterns in parallel. This method has the potential to provide an analysis of gene expression patterns at the genome level.

\section{Background}

In situ hybridisation (ISH) is the method of choice for describing the spatial expression pattern of a given gene. High resolution protocols provide cellular and even subcellular resolution. In multicellular organisms, ISH com- plements northern blotting, RT-PCR and microarrays, where the extraction of RNA from whole tissues invariably results in the loss of spatial information. Microarrays allow many genes to be studied in parallel and are currently one of the most powerful tools to study gene 
expression. However, microarray outputs often need to be verified by independent methods, such as ISH $[1,2]$, and because these downstream methods have a much lower capacity, verification is usually limited to one or a few genes. ISH must therefore be made more efficient and less time-consuming.

A number of variations on the traditional in situ protocols have been reported, including whole-mount ISH (WISH) [3], in situ PCR [4,5] and the use of vibratome sectioned tissues [6]. The main shortcoming of ISH is undoubtedly the low-throughput nature of the technique. In situ PCR (ISPCR) and RT-ISPCR are elegant techniques that can increase both sensitivity and throughput but they are at best only semi-quantitative [5] and it is desirable first to ascertain the expression pattern by conventional means in order to establish suitable conditions for each probe.

Efforts to make the ISH technique into a highly parallel, systematic process have been successful in flies and primitive chordates [7-9]. Attempts have been made to address this issue in plants using WISH and in situ PCR techniques $[10,11]$ although actual throughput remains undetermined.

High-throughput protocols used for animal embryos normally involve whole-mount methods [7,8,12], thus avoiding the need to section material. The challenges in applying similar techniques to plants include the large size of the tissues and the variable nature of the cell wall. These factors can variably compromise the penetration of probe and make microscopic examination more difficult and time-consuming. WISH is a possibility for Arabidopsis roots and seedlings [11], at least for low- and mediumthroughput. However, when performed on other larger tissues, such as seeds, WISH may require embedding and sectioning after the in situ has been performed to evaluate the results [13]. Therefore, the high-throughput advantages gained in the early stages of such procedures are effectively cancelled out.

Promoter fusions with reporter genes are another option for cellular localisation of transcripts but this approach has recognised shortcomings [14]. Elements controlling gene expression are known to be located not only in the traditional promoter region upstream of the coding region, but intergenically and, potentially, a considerable distance from the gene $[15,16]$. The resources required for mass transformation and the fact that not all plant species are amenable limits the application of this approach to well-studied model species.

As well as providing an independent means of screening genes for the desired expression profiles (differential expression, domain specific expression etc.) in gene dis- covery efforts, we envisage that high-throughput mRNA ISH is entirely feasible and will complement the evergrowing microarray data resources available [2,29] https:/ /www.genevestigator.ethz.ch/; $\quad$ http://www.weigel world.org/resources/microarray/AtGenExpress/. Recently real-time RT-PCR has been adapted for high-throughput processing [30]. While these approaches provide a wealth of expression data for functional genomics, they are unable to provide the spatial resolution that often directly reflects functional involvement in developmental processes. This inherent drawback in microarray technology has been elegantly addressed using cell sorting to isolate pure populations of a given cell type from the Arabidopsis root. However, this innovative approach is limited to species where suitable and diverse cell line markers are available [31]. It is further limited to tissues whose cells can be separated and sorted: roots are susceptible to protoplasting enzymes but shoots and many other tissues are not.

With these considerations in mind, we have deconstructed the "traditional" ISH protocol and developed a protocol for ISH that retains high resolution and specificity but integrates a degree of automation to a standardised and streamlined protocol. We have used wheat grain and Arabidopsis floral meristems as tests for this new highthroughput protocol and show that it is capable of highly parallel processing.

\section{Results and discussion Generating an integrated protocol}

One of the main challenges in mRNA ISH is developing an economical protocol that is applicable to large batches of different probes while maintaining a high level of specificity, sensitivity and resolution. The level of economy must be maintained throughout the process to provide a systematic high-throughput level of work. Figure 1 summarises the ISH protocol as described in previous reports $[17,18]$ and describes the five main components of the entire procedure. We have examined each of these components individually, but in context of the overall technique, with the view to (i) simplification, (ii) automation and (iii) optimisation. As a practical accompaniment to the following description we have included a step-by-step version of the protocol as used at the bench (Additional file $1)$.

\section{(i) Plant tissue preparation}

Plants grown under desired conditions were harvested, trimmed to allow penetration of solutions, and immediately fixed. These steps were carried out manually in both the standard and new protocols but, in order to automate the new protocol, the samples were placed in a Tissue-Tek Vacuum infiltration processor for further processing. 


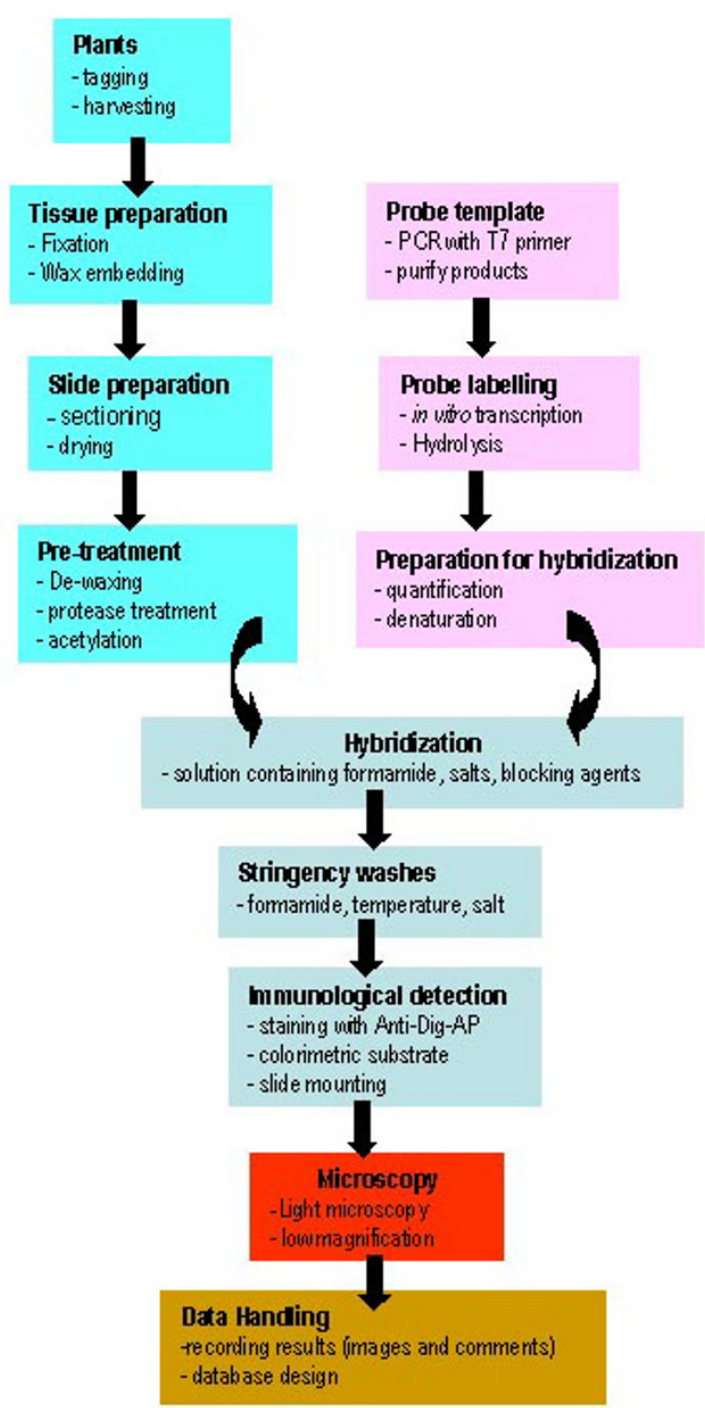

Figure I

Flowchart summarising mRNA in situ methodology.

The Tissue-Tek machine permits use of a combination of vacuum and pressure to exchange solutions at defined times and temperatures, relieving the operator of a large number of tedious steps as well as standardising the process. The initial fixation, dehydration and wax infiltration steps can take over a week to complete using the manual protocol, whereas the automated protocol reduces this to 24 hours. We evaluated various plant tissues prepared by both methods for tissue integrity and preservation of mRNA (signal strength). Arabidopsis tissues are equally well preserved and stained by both protocols but the automated procedure provided enhanced preservation in developing wheat seeds (data not shown). Many plant tis- sues, such as mature leaves, are very difficult to embed in wax using the manual protocol but even these recalcitrant tissues can be accommodated by altering the timing or pressure of processing.

Orientating and mounting in block for sectioning are skilled steps that normally require manual processing and these steps are therefore identical in both manual and automated protocols. Blocking up was carried out by hand to ensure favourable orientation but is facilitated by a dedicated embedding station. Sectioning cannot be automated due to the need to continuously assess section quality. However, the arrangement and number of tissue sections on the slide was made uniform using adherent, but removable, silicone isolators (Figure 2). This allowed the parallel screening of multiple probes on the same slide containing up to eight sections, each section in an isolated well.

When the sections had adhered to the slide, and the silicone isolators were removed, the slides were treated to remove wax and to make the sections receptive to the hapten-labelled probes. These down-stream treatments are universal to all tissues and appropriate for automation. However, these treatments are complex and we evaluated which ones were functional (i.e. produced an enhanced, yet specific signal) and which were redundant. Using a training set of probes and the traditional manual protocol, we systematically eliminated or reduced each step in the protocol and visually evaluated the final result. Slides were processed in parallel but with a proportion subjected to a protocol that omitted one or more steps normally used. This led to a reduction in the number of ethanol dehydration steps and elimination of the 're-fixation" step after proteinase K treatment. Some steps, although not absolutely essential, appeared to enhance the reliability of the process and these were retained: for example, acetic anhydride treatment was found to reduce background (especially on poly-lysine coated slides) and we increased the time allowed for de-waxing in xylene while applying agitation using the VP2000 slide processor (see below).

Finally, using a basic open-plan slide processor (VP2000), common in medical cytology labs, a reduced sectionprocessing protocol with essential steps only was automated and made completely hands-off. This also eliminates much of variation possible in a multi-step, closely timed procedure and led to more reproducible signals.

\section{(ii) Probe-making}

In the manual protocol, individual probes were made from linearized plasmids. This necessitates the analysis of each clone for suitable restriction sites. To eliminate timeconsuming individual analysis, we used a PCR strategy to produce linear plasmid inserts for probe transcription. 


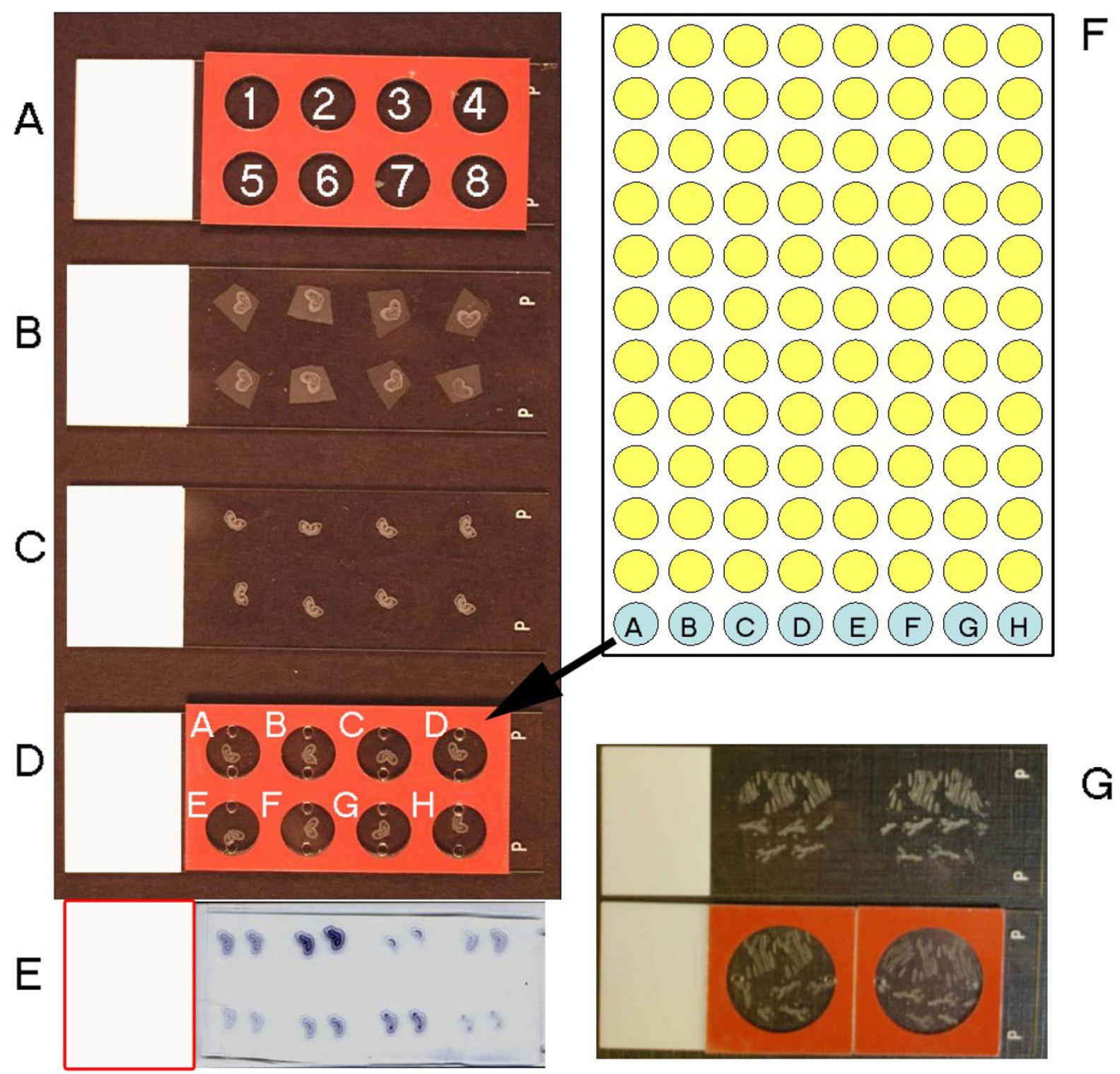

Figure 2

Arrangement of sections and organisation of probes: (A) Silicone isolators to position sections on slides. Positions are shown I to 8. (B) Sections placed in postions I to 8, dried and shown after removal of silicone isolator. (C) Sections after de-waxing step. (D) Sections in hybridisation chambers. A-H cross refers to plate position of the probes (F). (E) Sections after colour development. (F) Organisation of probes in 96 well plate. (G) Organisation of Arabidopsis sections showing larger format hybridisation chamber on lower slide.

This allows the production of large number of probes in parallel, particularly if the original clones are in a common vector. For a collection of genes inserted in the same orientation in a common vector, as found in most gene libraries, a common pair of primers can be designed to the flanking regions of the vector and used to prepare all 


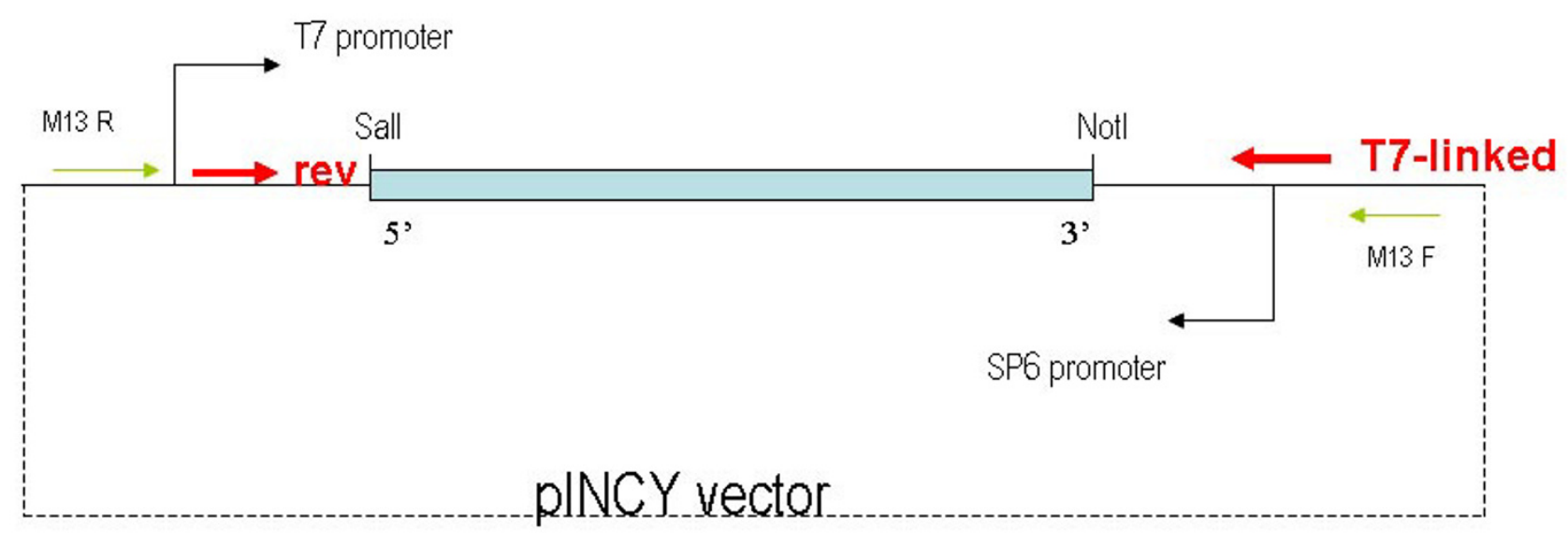

Figure 3

Schematic showing the production of templates for probe labelling. PCR reactions used cDNAs in pSPORT-derived vectors as template with a T7-linked 3' primer and a 5' primer based on vector sequence. T7 RNA polymerase was used to make all antisense probes.

probes. Thus, for the wheat cDNAs we designed a pair of primers against the flanks of the polylinker region for the pINCY vector (a derivative of the pSPORT vector from Invitrogen). The 3' primer contained a T7 RNA polymerase transcription site (see Figure 3). Thus, all the antisense probes were amplified using the same primer pair and subsequently all transcribed with T7 RNAP (T7 RNA Polymerase). This strategy was scalable: templates were produced in batches of 96 (using a liquid dispensing Qbot) and yield was estimated on a 96 well multi-slot E-gel (Invitrogen).

We also assessed the yield of different RNA polymerases. Although in vitro transcription is possible with any one of T7, T3 or SP6 RNAPs, T7 was undoubtedly the most efficient providing high yields of almost every cDNA. SP6 was found to be the least consistent, but could produce reasonable yields for a low proportion of clones. We did not investigate this further and routinely used T7 RNAP.

Next, we minimised the protocol for the in vitro labelling of the PCR products to the following essential steps only: transcription with dig-UTP (digoxigenin uridine triphosphate) for two hours, immediate hydrolysis for 30 minutes for all probes and immediate precipitation with ammonium acetate (which also neutralises the carbonate hydrolysis buffer) and ethanol for 30-60 minutes. The labelling procedure was performed in 96-well plate for- mat and took a total of four hours. To monitor transcription efficiency, a small aliquot from each batch of transcription products was run on an agarose gel to ensure that transcription is working and we repeated this test after the hydrolysis step.

The manual protocol describes individual hydrolysis times for each probe depending on the length of the DNA but we have found that once probes are hydrolysed to below a certain length, more detailed definition of an optimal probe size is not required. Hydrolysis fragments the probe and, in theory, the small fragments can access the RNA within tissue sections. However, subjecting the same transcript to several various hydrolysis incubations had little effect on signal strength. As template lengths ranged from 0.5 to $1.5 \mathrm{~kb}$, we subjected each labelled transcript to the same hydrolysis treatment. When assessed on an agarose gel, all probes produced small fragments within a comparable range. Labelled transcription products were resuspended in TE and could be stored at $-70^{\circ} \mathrm{C}$ where they remain usable for at least several months.

Accurate quantification of probes by dot-blot requires serial dilution but a simple qualitative test using a 1:100 dilution of each probe is usually sufficient to assess probe quality. If labelling is detected at this dilution, the same dilution in Hybridisation Solution (HS) can be denatured briefly and added to the slide for hybridisation. Since the 
probes are single stranded RNA, they are very prone to degradation during storage. Stability is much enhanced by diluting probes directly into the HS and storing them at $20^{\circ} \mathrm{C}$ : the HS contains $50 \%$ formamide which inactivates RNases.

\section{(iii) Hybridisation and signal detection}

At the end of the pre-treatment in the VP2000, the slides were dried and the silicone incubation chambers were carefully applied (Figure 2D). $40 \mu \mathrm{l}$ of HS/probe mix was applied to each chamber in a standardised order from 96 well plate to slide (i.e., plate A1-A8 to slide 1 etc.,) such that 96 probes (including controls) can be applied to 12 slides (Figure 2F). Hybridisation was overnight at a standard $50^{\circ} \mathrm{C}$ in a conventional incubator on horizontal slide racks.

Use of hybridisation chambers with the same dimensions as the isolator allowed at least 8 different probes to be applied to a single slide. A range of sizes of isolator and hybridisation chambers are available and different sizes may be better suited to different tissue types (Figure 2G). Use of glass coverslips for incubation steps in the manual protocol normally requires prolonged, but gentle, washing to remove coverslips without damaging the underlying sections. However, the chambers can be quickly removed and the slides were loaded immediately for a short washing step in the VP2000. In addition, we also evaluated the various post-hybridisation steps and found that the RNase treatment step could be omitted completely. The RNAse step is thought to decrease background staining in ISH but slides prepared with or without RNase are essentially identical.

After standard incubations in blocking solution and AntiDIG-AP (alkaline phosphatase) antibody, the slides were developed colorimetrically and a sample of the output is seen in Figure 2E. Using the colorimetric system rather than fluorescence means the progress of development can be monitored under a dissecting microscope and the problem of plant tissue autofluorescence is avoided. All reactions were stopped simultaneously, and all slides mounted permanently and could be stored for image-capture on the microscope.

\section{(iv) Processing of results}

Previous methods using one probe per slide meant that microscopic analysis was time-consuming and involved positioning many individual sections for optimal picture quality. In the automated protocol, with uniformly arranged sections representing multiple probes on every slide, image capture can be streamlined and has the potential for further automation.

\section{(v) Data collection and storage}

Images are collected in order and were directly linked to spreadsheet or database records of the probes used in the screening. In a recently published experiment on wheat seed development [19], batches of 96 probes were used to generate 288 images of 3 developmental stages. These images were labelled in order A1-96, B1-96 and C1-96 for each plate and stored accordingly. Representative results from the wheat project are shown in Figure 4A-F and are taken from different stages screened to show signal detection in the varied cell layers in the endosperm and surrounding tissues. These results illustrate that the automated protocol provides at least cellular resolution and in many cases, subcellular. Very specific patterns are defined within even thick-walled cells (such as the transfer cells of the nucellar projection and modified aleurone), which are likely to be recalcitrant to whole-mount procedures. Various other cell types are equally well stained with appropriate probes: the small cuboid cells of the young integument layers, the highly-vacuolate cells of the 9 DAA (days after anthesis) endosperm and the early multi-nucleate, but unicellular, coenocyte.

We also evaluated the protocol on other species, including Arabidopsis. Floral meristems were fixed and processed using the automated procedure and probes prepared in 96-well format. Using a training set of 4 previously characterised genes histone H4, AP3, AG and stm [18,20-22] (Figure 5) alongside genes encoding a variety of other cellular functions, we show that this approach has the potential for systematic spatial analysis of gene expression in a model organism, with at least cellular resolution (Figure $6)$. The expression patterns of a set of ten genes are shown in figure 6 includes some previously characterised genes including AtREM1, encoding a plant-specific regulatory protein and expressed in the floral meristems [23] (Figure 6A), CRABS CLAW, encoding a helix-loop-helix regulator of carpel development [24] (Figure 6J) and the recently described CORONA gene encoding a leucine zipper regulator of vascular tissue [25] (Figure 6K). These patterns are very similar to previously published results, indicating that the new protocol is robust and can be used for a range of genes without specific tailoring to each gene.

\section{Conclusion}

We describe a semi-automated system for highly parallel processing of ISH. We have introduced a substantial degree of automation to produce a system for performing high-throughput RNA-ISH on hundreds of plant tissue sections simultaneously without loss of resolution, specificity or sensitivity. This slide processing system has a capacity of at least 96 probes per week/per person for multiple developmental stages or experimental treatments and, with the exception of imaging, is scalable. Therefore, it is now feasible to contemplate genome-wide spatial 

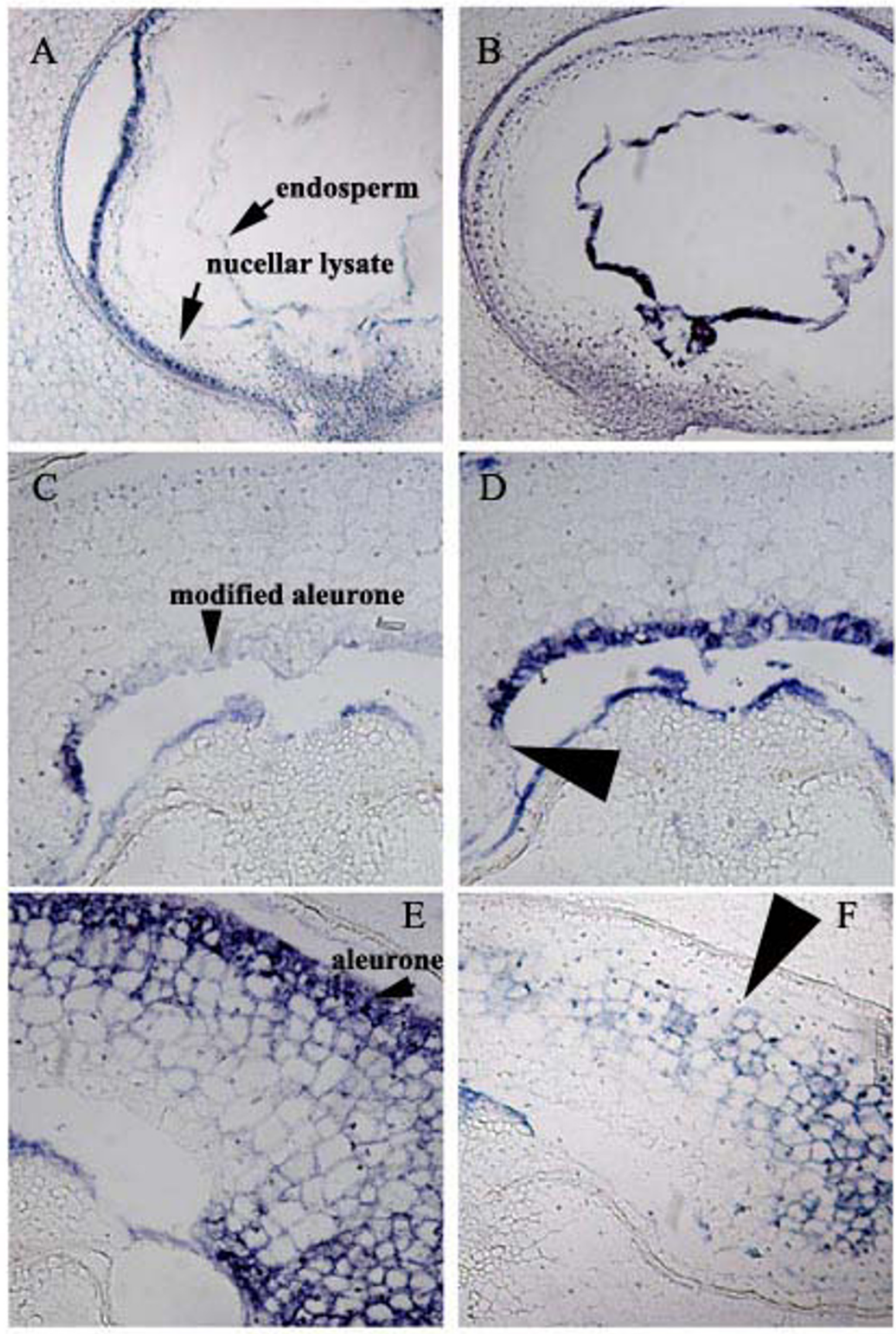

\section{Figure 4}

Examples of gene expression patterns in developing wheat grains. ID numbers indicate the Incyte gene code and can be used to search the wheat in situ database at SCRI http://bioinf.scri.sari.ac.uk/cgi-bin/insitu/home (user: guest; password: wheatinsitu).

(A) transcript detection specifically in the nucellar epidermis at 3DAA (ID 702007486). Arrowheads indicate no expression in the innermost nucellar lysate or the coenocytic endosperm. (B) coenocytic endosperm at 3DAA (ID 702038349) (C) peripheral cells of the modified aleurone only show transcript accumulation of an unknown gene at 9DAA (ID 701965703) (D) gene expression of a plantacyanin orthologue throughout the modified aleurone at 9DAA (ID 702044644). This section is from the same grain as in (C) and the arrowhead indicates that the most peripheral cells showing signal in (C) are not expressing plantacyanin. (E) a proteinase inhibitor is expressed strongly in the outer layers of the central endosperm at 9DAA (ID 701965839) $(F)$ in contrast to $(E)$, a gliadin storage protein gene is expressed throughout the central endosperm but not in the outermost layers (indicated with arrowhead; ID 702007003) 

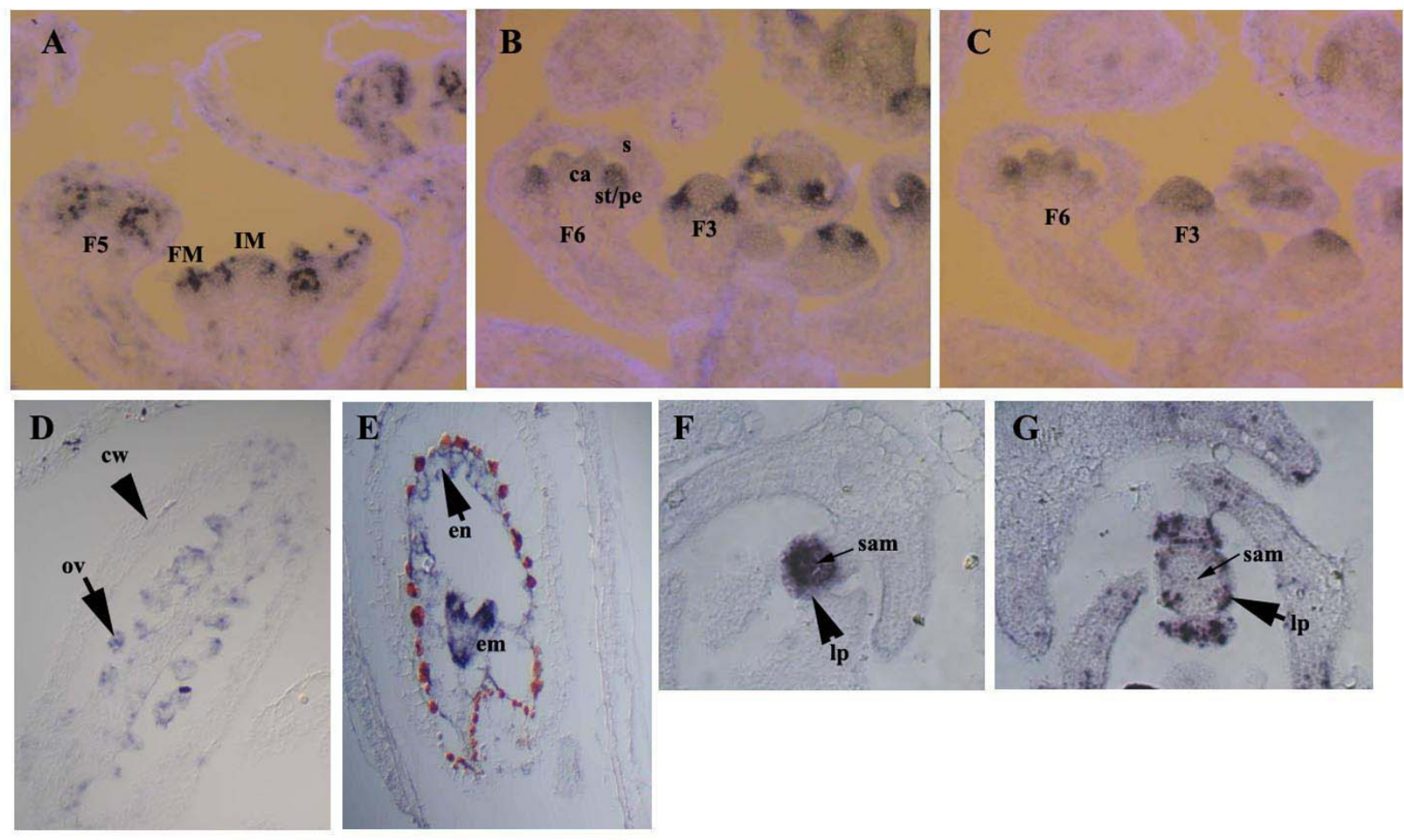

\section{Figure 5}

The automated ISH protocol on Arabidopsis tissues. Developing flowers (A-C), developing siliques (D), ovules (E) and transverse sections of the shoot apical meristem (SAM) of I0-day seedlings (F-G). Probes used are for histone $H 4$ (A, D, E, G), AP3 $(B), A G(C)$ and stm (F). (A-C) were counterstained with the cell wall dye, Calcofluor, which produces a light blue colour. Expression of $A P 3$ and $A G$ in serial sections (B and $C$ ) shows the distinct patterns of expression in the petal/stamen primordia for the class B AP3 gene and in the carpel primordia for the class C AG. In (D) arrows indicate expression of histone H4 in the developing ovules but by this stage there is no expression in the silique/carpel wall. Expression is also detectable in the endosperm of the developing ovule as well as in the cotyledons and root meristem of the embryo $(E)$. In $(F)$ an arrow indicates absence of stm expression in the leaf promordia but histone is expressed here and in the slightly older leaves (G). IM, inflorescence meristem; FM, floral meristem, number indicates the approximate flower stage; s, sepal; ca, carpel; st, stamen; pe, petal; cw, carpel wall; ov, ovule; en, endosperm; em, embryo; sam, shoot apical meristem; lp, leaf primordium.

analyses of gene expression at cellular resolution in both crop and model plant species.

To achieve genome-wide coverage in any species, certain prerequisites are necessary. First, sequences representing the expressed genes must be suitable for making probes and be available in an organized format. Second, the remaining manual steps must be further streamlined and, ideally, automated. Finally, automated image collection, analysis and quantification methods need to be developed.
Several projects are currently underway whose collective aim is to provide the expressed Arabidopsis genome as organized libraries of clones. Thus, a large proportion of Arabidopsis genes are currently available as trimmed ORFs (open reading frames) from SALK http://signal.salk.edu/ cdnastatus.html and ESTs (expressed sequence tags) from ABRC (Arabidopsis Biological Resource Centre) http:www.biosci.ohio-state.edu/ plantbio/Facilities/ abrc/abr chome.htm, or as 3' UTRs (untranslated regions) or as groups of structurally related genes from special projects such as REGIA (Regulatory Gene Initiative in Arabidopsis) http://www.epsoweb.org/catalog/EU/fp5/ REGIA.htm. These collections tend to present genes in a 

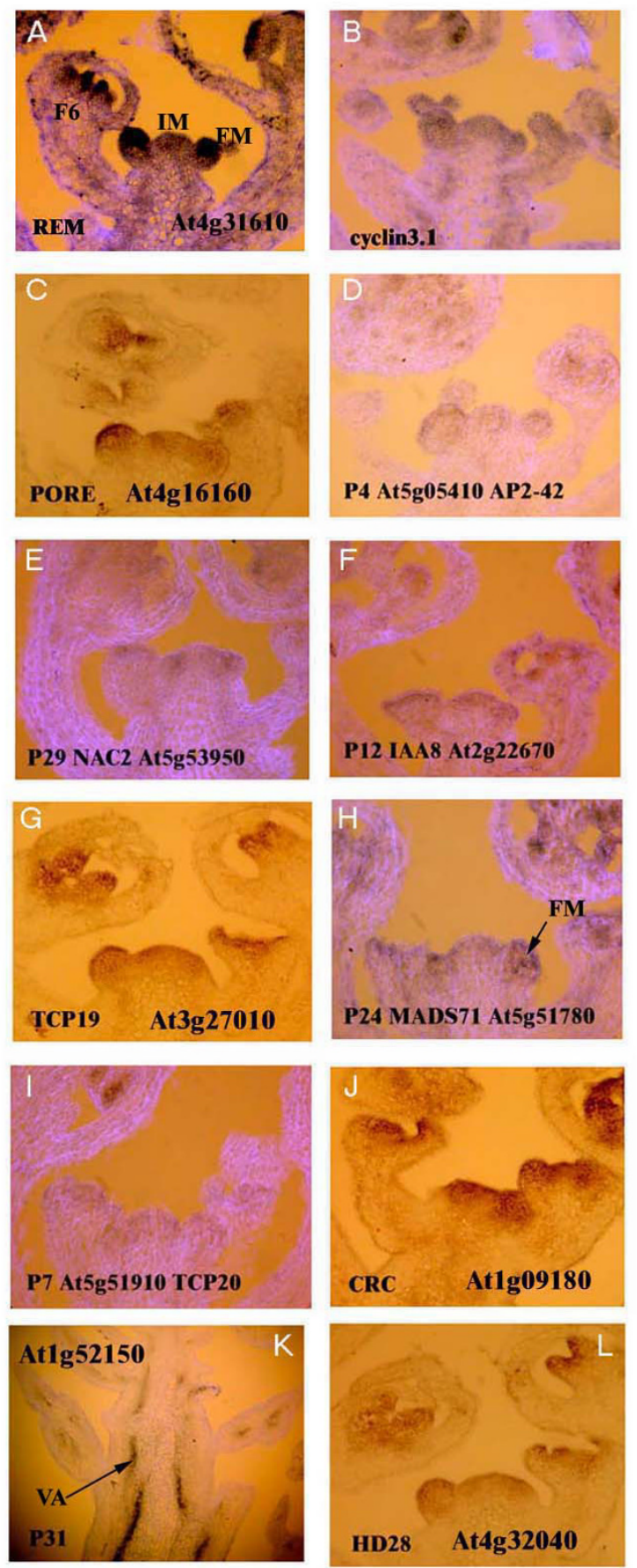

\section{Figure 6}

A selection of gene expression patterns (mostly transcription factors) in young Arabidopsis flowers (A-L). AGI gene annotations accompany each figure in the panel. Expression in young floral meristems is strong in $A, B, C, G, J$ and $L$. The arrow in $\mathrm{H}$ indicates weaker but specific expression in the floral meristem and in $K$ there is strong expression of CNA in the vasculature (VA). A, B D, E, F, H, and I have been counterstained with the cell wall stain, Calcofluor, which produces a light blue colour. consistent format that is amenable to automation. In most crop species, where the genomes are as yet incomplete, large EST collections are available. However, even these can provide useful information when used in conjunction with microarrays, where gene expression data can be confirmed and resolved to individual cell types and tissue layers [19].

Throughput is somewhat lower in Arabidopsis than in wheat, largely due to the time required for sectioning the smaller and more heterogeneous tissues. The proportion of usable sections is less and the tissue complexity requires greater imaging times. However, the smaller size of Arabidopsis facilitates whole mount approaches [3,11] and, used with confocal microscopy and fluorescent imaging, this could completely eliminate manual sectioning without compromising either resolution or throughput.

Further automation is therefore required. Recent developments in slide processing have provided improved and more cost-effective slide processors that automate all the steps from de-waxing to mounting, including hybridisation (unpublished results) but imaging and analysis remain significant rate-limiting factors. Automated imaging and analysis involving machine-learning, are essential to extend this approach to the analysis of whole genomes. Such approaches have been developed for the analysis of tissue microarrays in the analysis of protein expression in various cancers, though these technologies still involve protein immunohistochemisty more than mRNA in situ hybridization [26-28]. However, as they employ a similar colour-based detection system, the technology should be transferable.

\section{Methods \\ Preparation of plant material}

Wheat plants (variety Savannah) were grown under controlled environment conditions $\left(16^{\circ} \mathrm{C}, 16 \mathrm{~h}\right.$ light $)$ and ears tagged daily at anthesis. Arabidopsis Col-0 was grown in glasshouse under a 16-hour light regime. Wheat grains harvested at 3,6 and 9 DAA were trimmed and Arabidopsis floral meristems were removed just after bolting. All tissues were fixed in $4 \%$ paraformaldehyde, then transferred to the Tissue Tek VIP (Vacuum Infiltration Processor, Sakura) for an automated fixation/dehydration/infiltration process as follows: fixative $6 \mathrm{~h} 35^{\circ} \mathrm{C} ; 70 \%$ ethanol 1 h $35^{\circ} \mathrm{C}, 80 \%$ ethanol $1.5 \mathrm{~h} 35^{\circ} \mathrm{C}$; $90 \%$ ethanol $2 \mathrm{~h} 35^{\circ} \mathrm{C}$; $100 \%$ ethanol $1 \mathrm{~h} 35^{\circ} \mathrm{C} ; 100 \%$ ethanol $1.5 \mathrm{~h} 35^{\circ} \mathrm{C}$ (repeat for $2 \mathrm{~h}$ ); xylene $0.5 \mathrm{~h} 35^{\circ} \mathrm{C}$ (repeat for $1 \mathrm{~h}$ and again for $1.5 \mathrm{~h}$ ); wax $1 \mathrm{~h} 60^{\circ} \mathrm{C}$ (repeat once, then again twice for $2 \mathrm{~h}$ ). All steps were performed under vacuum. Samples were then transferred to the Tissue Tek Embedding Console for embedding in paraffin wax. 


\section{Section preparation}

Sections $(14 \mu \mathrm{m})$ from wheat samples at the required stages and $8 \mu \mathrm{m}$ sections from floral meristems were cut on a Leica Microtome (RM2125RT) and ordered on polysine slides containing the silicone isolators (Grace Biolabs). After drying down at $42^{\circ} \mathrm{C}$ overnight suitable sections were selected for pretreatment.

Pretreatment steps were performed using the VP2000 Slide Processor (Vysis) using the following program: xylene $20 \mathrm{~min}$ (twice); $100 \%$ ethanol $10 \mathrm{~min}$, then through a 95\%, 85\%, 50\%, 30\% ethanol series (2 min each), PBS ( $3 \mathrm{mM} \mathrm{NaH}_{2} \mathrm{PO}_{4}, 7 \mathrm{mM} \mathrm{Na}_{2} \mathrm{HPO}_{4}, 130 \mathrm{mM}$ $\mathrm{NaCl})$ 3-4 min; proteinase $\mathrm{K}(2-3 \mu \mathrm{g} / \mathrm{ml}$ in $100 \mathrm{mM}$ Tris, $10 \mathrm{mM}$ EDTA pH7.5) $30 \mathrm{~min}$ at $37^{\circ} \mathrm{C}$; glycine $(0.2 \%) 2$ min; PBS 3-4 min; acetic anhydride $(0.5 \%$ in $0.1 \mathrm{M}$ triethanolamine $\mathrm{pH}$ 8) $10 \mathrm{~min}$; PBS 3-4 min, then back through the ethanol series. Slides were completely dry at this stage and could be stored at $4^{\circ} \mathrm{C}$ until hybridisation.

\section{Generating templates and labeling probes}

Wheat cDNAs for screening are supplied as inserts in a vector derived from pSPORT1. Primers were designed in order to append a T7 RNAP site to the 3' end of the insert with the other primer nested inside the native vector T7 RNAP site; T7.2 5' GAATTGTAATACGACTCACTATAGGGCCAGTGAATTGAATTTAGG $3^{\prime}$ and R7.2 5'AGGGAAAGCTGGTACGCCTGC 3' (T7 RNAP promoter binding site underlined). Arabidopsis histone $\mathrm{H} 4$ was amplified from pBluescript and AP3, AG, REM from pGEM vectors with universal forward and reverse primers for subsequent transcription with T7 RNAP. PCR reactions were performed with the following cycle: $94^{\circ} \mathrm{C} 3 \mathrm{~min}$, then 30 cycles of $94^{\circ} \mathrm{C} 45 \mathrm{~s}, 63^{\circ} \mathrm{C} 45 \mathrm{~s}$ and $72^{\circ} \mathrm{C} 1.5 \mathrm{~min}$, final extension of $72^{\circ} \mathrm{C}$ for $6 \mathrm{~min}$. For 96-well plates PCRproduct purification was done using the Montage Cleanup Kit (Millipore).

In vitro transcription was performed in $10 \mu \mathrm{l}$ reactions for $2 \mathrm{~h}$ at $37^{\circ} \mathrm{C}$ in the presence of digoxigenin-UTP (DigUTP)-nucleotides $(0.35 \mathrm{mM})$. Hydrolysis was carried out immediately in $100 \mathrm{mM}$ carbonate buffer $\mathrm{pH} 10.2$ at $60^{\circ} \mathrm{C}$ for $30 \mathrm{~min}$, and products precipitated in $2.5 \mathrm{M}$ ammonium acetate and $3 \mathrm{vol}$ absolute ethanol for $1 \mathrm{~h}$ at $4{ }^{\circ} \mathrm{C}$. Plates were centrifuged at $4000 \mathrm{rpm}$ for $30 \mathrm{~min}$ and pellets resuspended in $30 \mu \mathrm{l}$ TE $(100 \mathrm{mM}$ Tris, $10 \mathrm{mM}$ EDTA) buffer. Dilutions $(100 \mathrm{x})$ were made in water and $1 \mu \mathrm{l}$ of each spotted on nitrocellulose for dot-blot: $30 \mathrm{~min}$ in blocking solution (Sigma), $30 \mathrm{~min}$ in anti-DIG-alkaline phosphatase (Roche); 5 min wash in TBS (10 mM Tris, $250 \mathrm{mM} \mathrm{NaCl}$ ); $5 \mathrm{~min}$ in AP-buffer (100 mM Tris, 100 $\mathrm{mM} \mathrm{NaCl} \mathrm{pH} \mathrm{9.5;} 50 \mathrm{mM} \mathrm{MgCl}_{2}$ ) and developed as described above until signal was sufficient. All probes were then diluted 100-fold in hybridisation solution (300 $\mathrm{mM} \mathrm{NaCl}, 10 \mathrm{mM}$ Tris $\mathrm{pH}$ 6.8, $10 \mathrm{mM} \mathrm{NaPO}_{4}, 5 \mathrm{mM}$
EDTA, 50\% formamide, 5\% dextran sulphate, $0.5 \mathrm{mg} / \mathrm{ml}$ tRNA, $1 \times$ Denhardt's, $0.1 \mathrm{mg} / \mathrm{ml}$ salmon testis DNA) and maintained stably at $-20^{\circ} \mathrm{C}$ until hybridization.

\section{Hybridisation and washing}

Chambers (Grace Biolabs) were applied securely to the slides (after pre-treatment) and probes (diluted in hybridisation solution) were applied to one well ( 2 sections) for the 3 stages individually. Coverslips were placed on the chambers to prevent evaporation and hybridisation was performed overnight in a $50^{\circ} \mathrm{C}$ incubator.

Chambers were removed and slides arranged in the VP2000 for washing program: $15 \mathrm{~min}$ in $2 \times \operatorname{SSC}(0.3 \mathrm{M}$ $\mathrm{NaCl}, 0.03 \mathrm{M} \mathrm{Na}$ citrate), $50 \%(\mathrm{v} / \mathrm{v})$ formamide at $40^{\circ} \mathrm{C}$; $40 \mathrm{~m}$ in the same at $50^{\circ} \mathrm{C} ; 20 \mathrm{~min}$ in $1 \times \mathrm{SSC}, 50 \%(\mathrm{v} / \mathrm{v})$ formamide at $50^{\circ} \mathrm{C}$ (all steps with constant agitation); 5 min in $1 \times$ SSC at room temperature; $5 \mathrm{~min}$ in $1 \times$ TBS at room temperature. Then slides were transferred into trays for staining: 1\% blocking solution (Roche) in TBS $1 \mathrm{~h}, 1 \times$ TBS containing $1 / 3000$ dilution of Anti-DIG AP and $0.05 \%(\mathrm{v} / \mathrm{v})$ Tween-20 $1 \mathrm{~h} ; 4 \times 10 \mathrm{~min}$ washes in $1 \times \mathrm{TBS}$; $5 \mathrm{~min}$ in AP-Buffer $(0.1 \mathrm{M}$ Tris, $0.1 \mathrm{M} \mathrm{NaCl}, 50 \mathrm{mM}$ $\left.\mathrm{MgCl}_{2}\right)$; developed in AP-Buffer containing NBT $(0.1 \mathrm{mg} /$ $\mathrm{ml})$ and BCIP $(0.075 \mathrm{mg} / \mathrm{ml})$ for a maximum of $24 \mathrm{~h}$. Slides were then washed several times in water to stop the reaction followed by sequential washes in $70 \%$ and $100 \%$ ethanol to remove excess stain (the duration of the ethanol washes depends on the level of colour development which was monitored by eye). Slides were then allowed to dry and permanently mounted in Entellan (Merck).

\section{Image capture and analysis}

One section for each stage for each probe screened was photographed on a Nikon E800 microscope using a digital camera under brightfield conditions for wheat sections and with UV filter for the calcofluor-counterstained Arabidopsis sections. Images were recorded sequentially as ordered on the slides. Magnifications and camera settings remained unchanged for all images through all stages for wheat section and likewise for floral meristems.

\section{Competing interests}

The author(s) declare that they have no competing interests.

\section{Authors' contributions}

SD developed the system described, performed all work for the wheat experiments and wrote the manuscript; JC and $\mathrm{BC}$ provided technical assistance on adapting the technique to Arabidopsis; JHD, PS and LD were co-supervisors; JHD co-wrote the manuscript. 


\section{Additional material}

\section{Additional File 1}

Detailed high-throughput in situ hybridisation protocol. An illustrated text document is attached with a protocol written in step by step detail for people working at the bench.

Click here for file

[http://www.biomedcentral.com/content/supplementary/17464811-1-8-S1.doc]

\section{Acknowledgements}

The authors wish to thank Sarah Collier and Mary Byrne John Innes Centre) for expertise working with Arabidopsis; Cathie Martin, Jan Traas, and Robert Sablowski for probes; Steve Evans and Gawain Bennett (Syngenta) for help with the wheat probe preparation; David Leader, Wolfgang Schuch and Simon Bright for facilitating the collaboration with Syngenta and the BBSRC, Syngenta and the John Innes Centre for funding.

\section{References}

I. Chuaqui RF, Bonner RF, Best CJ, Gillespie JW, Flaig MJ, Hewitt SM Phillips JL, Krizman DB, Tangrea MA, Ahram M, Linehan WM, Knezevic V, Emmert-Buck MR: Post-analysis follow-up and validation of microarray experiments. Nature Genetics 2004 32:509-5।4

2. Wellmer F, Riechmann JL, Alves-Ferreira M, Meyerowitz EM: Genome-wide analysis of spatial gene expression in Arabidopsis flowers. Plant Cell 2004, I 6: I3|4-1326.

3. Almeida Engler J, de Van Montagu M, Engler G: Whole-mount in situ hybridization in plants. Methods Mol Biol 1998, 82:373-384.

4. Johansen B: In Situ PCR on Plant Material with Sub-cellular Resolution. Ann Bot 1997, 80:697-700.

5. Pesquet E, Barbier O, Ranocha P, Jauneau A, Goffner D: Multiple gene detection by in situ RT-PCR in isolated plant cells and tissues. Plant J 2004, 39:947-59.

6. Borlido J, Pereira S, Ferreira R, Coelho N, Duarte P, Pissarra J: Simple and Fast In Situ Hybridization. Plant Mol Biol Rep 2002, 20:219-229.

7. Tomancak P, Beaton A, Weiszmann R, Kwan E, Shu S, Lewis SE, Richards S, Ashburner M, Hartenstein V, Celniker SE, Rubin GM: Systematic determination of patterns of gene expression during Drosophila embryogenesis. Genome Biol 2002, 3:RESEARCH0088-8.

8. Butler MJ, Jacobsen TL, Cain DM, Jarman MG, Hubank M, Whittle JR, Phillips $R$, Simcox A: Discovery of genes with highly restricted expression patterns in the Drosophila wing disc using DNA oligonucleotide microarrays. Development 2003, 130:659-70.

9. Satou Y, Takatori N, Fujiwara S, Nishikata T, Saiga H, Kusakabe T, Shin-i T, Kohara $Y$, Satoh N: Ciona intestinalis cDNA projects: expressed sequence tag analyses and gene expression profiles during embryogenesis. Gene 2002, 287:83-96.

10. Koltai H, McKenzie Bird D: High Throughput Cellular Localization of Specific Plant mRNAs by Liquid-Phase in Situ Reverse Transcription-Polymerase Chain Reaction of Tissue Sections. Plant Physiol 2000, I 23:1203-1212.

II. Friml J, Benkova E, Mayer U, Palme K, Muster G: Automated whole mount localisation techniques for plant seedlings. Plant $] 2003$ 34:115-124.

12. Tautz $D$, Pfeifle $C$ : A non-radioactive in situ hybridization method for the localization of specific RNAs in Drosophila embryos reveals translational control of the segmentation gene hunchback. Chromosoma 1989, 98:81-85.

13. Reid AJ, Rogers AC, Greenwood JS: Rapid plastic embedding is compatible with colorimetric detection following whole mount in situ hybridization in plant specimens. Biotechnic and Histochemistry 200I, 76:3-I0.

14. Taylor C: Promoter Fusion Analysis: An Insufficient Measure of Gene Expression. Plant Cell 1997, 9:273-275.
15. Sieburth LE, Meyerowitz EM: Molecular dissection of the AGAMOUS control region shows that cis elements for spatial regulation are located intragenically. Plant Cell 1997, 9:355-365.

16. Larkin JC, Oppenheimer DG, Pollock S, Marks MD: Arabidopsis GLABROUSI gene requires downstream sequences for function. Plant Cell 1993, 5:1739-1748.

17. Coen ES, Romero JM, Doyle S, Elliott R, Murphy G, Carpenter R: floricaula: a homeotic gene required for flower development in antirrhinum majus. Cell 1990, 63:13II-22.

18. Fobert PR, Coen ES, Murphy GJ, Doonan JH: Patterns of cell division revealed by transcriptional regulation of genes during the cell cycle in plants. $E M B O \mid$ 1994, | 3:6 |6-24

19. Drea S, Leader DJ, Arnold BC, Shaw P, Dolan L, Doonan JH: Systematic spatial analysis of gene expression during wheat caryopsis development. Plant Cell 2005, 17:2172-2I85.

20. Jack T, Brockman LL, Meyerowitz EM: The Homeotic Gene APETALA3 of Arabidopsis thaliana Encodes a MADS Box and is Expressed in Petals and Stamens. Cell 1992, 68:683-697.

21. Bowman JL, Drews GN, Meyerowitz EM: Expression of the Arabidopsis Floral Homeotic Gene AGAMOUS is Restricted to Specific Cell Types Late in Flower Development. Plant Cell I991, 3:749-758.

22. Long J, Barton MK: Initiation of axillary and floral meristems in Arabidopsis. Dev Biol 2000, 21 8:34I-353.

23. Franco-Zorrilla JM, Cubas P, Jarillo JA, Fernandez-Calvin B, Salinas J, Martinez-Zapater JM: AtREMI, a member of a new family of B3 domain-containing genes, is preferentially expressed in reproductive meristems. Plant Physiol 2002, I 28:4| 8-427.

24. Bowman JL, Smyth DR: CRABS CLAW, a gene that regulates carpel and nectary development in Arabidopsis, encodes a novel protein with zinc finger and helix-loop-helix domains. Development 1999, 1 26:2387-2396.

25. Green KA, Prigge MJ, Katzman RB, Clark SE: CORONA, a Member of the Class III Homeodomain Leucine Zipper Gene Family in Arabidopsis, Regulates Stem Cell Specification and Organogenesis. Plant Cell 2005, 1 7:691-704.

26. Camp RL, Chung GG, Rimm DL: Automated subcellular localization and quantification of protein expression in tissue microarrays. Nat Med 2002, 8: $1323-1327$.

27. Brey EM, Lalani Z, Johnston C, Wong M, Mclntire LV, Duke PJ, Patrick CW Jr: Automated selection of DAB-labeled tissue for immunohistochemical quantification. J Histochem Cytochem 2003, $51: 575-584$

28. Fernandez DC, Bhargava R, Hewitt SM, Levin IW: Infrared spectroscopic imaging for histopathologic recognition. Nat Biotechnol 2005, 23:469-474.

29. Alba R, Fei Z, Payton P, Liu Y, Moore SL, Debbie P, Cohn J, D'Ascenzo M, Gordon JS, Rose JK, et al.: ESTs, cDNA microarrays, and gene expression profiling: tools for dissecting plant physiology and development. Plant / 2004, 39:697-7/4

30. Czechowski T, Bari RP, Stitt M, Scheible WR, Udvardi MK: Realtime RT-PCR profiling of over 1400 Arabidopsis transcription factors: unprecedented sensitivity reveals novel rootand shoot-specific genes. Plant J 2004, 38:366-79.

31. Birnbaum K, Shasha DE, Wang JY, Jung JW, Lambert GM, Galbraith DW, Benfey PN: A gene expression map of the Arabidopsis root. Science 2003, 302:1956-60.

Publish with BioMed Central and every scientist can read your work free of charge

"BioMed Central will be the most significant development for disseminating the results of biomedical research in our lifetime. "

Sir Paul Nurse, Cancer Research UK

Your research papers will be:

- available free of charge to the entire biomedical community

- peer reviewed and published immediately upon acceptance

- cited in PubMed and archived on PubMed Central

- yours - you keep the copyright
BioMedcentral 